\title{
Medicalización de la vida (II)
}

Ramón Orueta Sánchez ${ }^{\mathrm{a}}$, Coral Santos Rodríguez ${ }^{\mathrm{b}}$, Enrique González Hidalgoc, Eva Ma Fagundo Becerra ${ }^{c}$, Gemma Alejandre Lázaro ${ }^{a}$, Javier Carmona de la Morena ${ }^{a}$, Javier Rodríguez Alcaláa ${ }^{a}$ José $\mathrm{M}^{\mathrm{a}}$ del Campo del Campo ${ }^{\mathrm{b}}$, María Luisa Díez Andrés ${ }^{\mathrm{d}}$, Natalia Vallés Fernández ${ }^{\mathrm{a}}$, Teresa Butrón Gómez ${ }^{\mathrm{d}}$

a Médico de Familia, Toledo.

${ }^{\mathrm{b}}$ Médico de Familia, Albacete.

cMédico de Familia,

Ciudad Real.

'Médico de Familia,

Guadalajara.

Correspondencia: Ramón Orueta Sánchez, Centro de Salud Sillería, C/ Sillería s/n 45001 - Toledo.

E -mail: roruetas@gmail.com.

Recibido el 5 de mayo de 2011.

Aceptado para su publicación el 12 de mayo de 2011.

\section{RESUMEN}

La medicalización de la vida es uno de los problemas que actualmente contribuyen a la masificación de las consultas del Médico de Familia y de los Servicios de Urgencias, provocando, a la vez, dificultades para proporcionar una atención de alta calidad y frustración en una buena parte de los profesionales. Entendemos por medicalización el proceso de convertir situaciones que han sido siempre normales en cuadros patológicos y pretender resolver, mediante la medicina, situaciones que no son médicas, sino sociales, profesionales o de las relaciones interpersonales. Los profesionales sanitarios son, a la vez, actores y victimas de dicho proceso. La medicalización tiene como principales consecuencias la trasformación de personas sanas en enfermos, el aumento de daños iatrogénicos, el consumo de recursos sanitarios y la pérdida de eficacia y eficiencia de los mismos. Entre las actuaciones recomendadas cabe destacar acciones encaminadas a regular las expectativas de la población, delimitar el campo de acción de la medicina, fomentar el autocuidado y actuar de acuerdo con las evidencias sanitarias existentes. Palabras clave. Servicios de Salud, Procedimientos Innecesarios, Actitud frente a la Salud.

\section{ABSTRACT}

Medicalisation of every-day life (II)

Medicalisation of every-day life is one of the problems currently contributing to the massification of visits to Doctors' surgeries or Hospital Emergency Departments, thus making it difficult to provide high quality healthcare and causing frustration for many health professionals. Medicalisation is understood as being the process of turning normal human conditions into medical conditions or diseases and trying to resolve them through medicines. Such conditions are not medical, but social or professional conditions or issues related to interpersonal relationships. Health professionals are both the actors and the victims of this process. The main consequences of medicalisation are: transforming healthy persons into patients, increasing iatrogenic harm and the consumption of healthcare resources and loss of efficacy and efficiency of the same. Amongst the recommended actions we emphasise those directed towards regulating the expectations of the population, restricting the field of action of medicine, encouraging self-care and acting according to current healthcare evidence.

Key words. Health Services Misuse, Unnecessary Procedures, Attitude to Health.

\section{CONSECUENCIAS}

Las actuaciones médicas pueden ocasionar daño o perjuicio tanto por omisión como por acción de las mismas, representando la medicalización un caso de daño potencial por acción. Siempre debe ser tenida en cuenta la premisa de que toda actuación no fundamentada es iatrogénica, ya sea desde el punto de vista médico, social o cultural, y la medicalización es un ejemplo claro de ello, que se traduce en múltiples y muy diversas consecuencias. Debe mencionarse, así mismo, que muchas de las consecuencias de la medicalización son a la vez causas de la misma, contribuyendo a su crecimiento y perpetuación.

\section{Consecuencias para la sociedad}

- Medicalización de la vida. La primera consecuencia de la medicalización es el aumento progresivo de dicha medicalización. Es una rueda que 
nunca tiene fin y que se retroalimenta; cada vez se trasforman en enfermedades más situaciones/ problemas no patológicos y esto a su vez contribuye a que se creen nuevas enfermedades y nuevos enfermos.

- Percepción de peor salud que nunca. La sociedad se encuentra en la paradoja de que en el momento que mayor es el nivel de salud objetivo existente y cuando mayor es el número de servicios sanitarios prestados, peor es la sensación de salud subjetiva y mayor es la demanda de servicios sanitarios. Ello es debido fundamentalmente a que no se ven cumplidas las expectativas existentes, creadas de forma artificiosa, al no existir soluciones para todo tipo de problemas y/o situaciones.

- "Sanitarización" de la sociedad. La sociedad se hace cada vez más dependiente del sistema sanitario y se requiere la participación de sus profesionales en todos los aspectos de la vida (enfermeras en los colegios, certificados médicos para ir a un balneario, etc.).

- Aumento de enfermedades/problemas de salud iatrogénicos. Entendiendo como tales a aquellos procesos que no se habrían producido si no se hubieran aplicado recomendaciones, pruebas diagnósticas o tratamientos farmacológicos o quirúrgicos. El uso irreflexivo de los recursos sanitarios disponibles ocasiona efectos perjudiciales graves. Cuantas más intervenciones se realizan (preventivas, diagnósticas 0 terapéuticas), aunque sea con elevados niveles de calidad, mayor es la morbimortalidad. Por otra parte, al pensar en iatrogenia no debemos olvidar, además de los efectos indeseables puramente somáticos, aquellos otros menos tangibles como pueden ser el efecto de ser "etiquetado como enfermo" o la ansiedad que puede generar un falso positivo de una prueba de cribado.

- Aumento del consumo de fármacos. En estrecha relación con el punto anterior, la medicalización ocasiona un aumento del consumo de medicamentos, en muchas ocasiones innecesarios $y / 0$ ineficaces, que aumenta el riesgo de reacciones adversas, interacciones etc.

- Sentimiento de frustración. Cuando se llevan a cabo actuaciones no eficaces, estas no obtienen los resultados esperados por la población y este incumplimiento de expectativas puede acabar generando frustración, desconfianza o ansiedad, que a su vez provocan la búsqueda de nuevas soluciones en el sector sanitario fomentando nuevas medicalizaciones.
- Disminución de la competencia individual. La búsqueda de soluciones externas (generalmente el sector sanitario) a todo tipo se situaciones conlleva una pérdida progresiva de las formas no médicas de afrontar las dificultades y de la capacidad de autocuidados.

- Disminución de los recursos informales. Aunque la medicalización de la vida no es el factor principal de la disminución de los distintos recursos informales en la sociedad, sí que es un factor influyente en dicha situación, al dar prioridad a la atención prestada por los profesionales sobre los prestados por dichos recursos.

\section{Consecuencias para las Instituciones y los profesionales}

- Sistema sanitario desbordado. La existencia de nuevas demandas, de demandas repetidas por el mismo cuadro, la petición de mayor número de pruebas complementarias, etc. está generando un sistema cada vez más masificado y con listas de espera desorbitadas o con una disminución de la calidad de la atención. En Atención Primaria genera la masificación de las consultas, lo que impide dedicar el tiempo necesario para cada paciente, contribuyendo a aumentar la insatisfacción del paciente y la inseguridad del profesional. Estas circunstancias, a su vez, generan nuevas consultas, solicitud de mayor número de pruebas, más tratamientos ineficaces... más medicalización. En Atención Hospitalaria genera la existencia de listas de espera crecientes, lo que conlleva situaciones desafortunadas que llegan a la opinión pública, generando mayor insatisfacción y descrédito del sistema.

- Aumento del gasto sanitario. La incorporación a la cartera de servicios de situaciones que responden a necesidades demandadas pero no reales y la incorporación al ámbito sanitario de situaciones anteriormente atendidas por otros sectores generan aumento del gasto sanitario. También contribuyen a aumentar dicho gasto situaciones más cercanas a los clínicos como el incremento irracional del empleo de nuevas tecnologías o la prescripción de tratamientos ineficaces. Este aumento del gasto sanitario actúa en detrimento de otros sectores que pueden contribuir positivamente al estado de bienestar.

- Pérdida de efectividad y equidad del sistema. El aumento del gasto sanitario comentado en el apartado anterior, además de ir en detrimento de los recursos destinados a otros sectores, también 
ocasiona que actuaciones de probada eficacia se vean marginadas o retrasadas en el tiempo. Como consecuencia puedan verse relegadas personas con necesidades reales y para las que si existen actuaciones eficaces. Desde otra perspectiva, la demora en la realización de pruebas diagnósticas, intervenciones quirúrgicas, etc. genera también pérdida de equidad, porque las personas con mayores recursos pueden utilizar sistemas paralelos (medicina privada) para la solución de sus problemas.

- Deshumanización de la atención. Muchos de los factores relacionados con la medicalización de la vida, como la parcelación de la atención, la superespecialización de los profesionales, la tecnificación del sistema, etc., son factores que están contribuyendo a que la atención sanitaria vaya perdiendo una faceta esencial de la misma como es el componente humano.

- Transformación de factores de riesgo en enfermedades. La realización indiscriminada de actividades de cribado, las revisiones repetidas por factores de riesgo, etc. acaban convirtiendo a personas sanas en enfermos dependientes del sistema.

- Aumento de iatrogenismo. Ya comentado en el apartado dedicado a las consecuencias para la población.

- Alteración de la relación profesional-paciente. Los pacientes se ven defraudados al no obtener los resultados esperados y no cumplirse sus expectativas; los profesionales reciben demandas de atención para las que no están preparados para dar respuesta, al quedar fuera de su ámbito de actuación, y todo ello altera la relación entre la población y los profesionales. Una forma de valorar este aspecto es a través del aumento de las reclamaciones existentes hacia el sector sanitario tanto de manera formal (judiciales) como informal (reclamaciones, denuncias en medios de comunicación, etc.).

- Insatisfacción de los profesionales. Al igual que los pacientes sienten frustración por no ver satisfechas sus expectativas, los profesionales tampoco se encuentran satisfechos con su trabajo. El síndrome de desgaste profesional o Burnout, que quizás represente otra forma de medicalización, es una expresión de esta insatisfacción.

A modo de conclusión, todas estas consecuencias comentadas de la medicalización de la vida pueden ser resumidas en la siguiente frase de Moynihan: "la medicalización de la vida es un proceso que convierte a personas sanas en enfermas, que produce daño iatrogénico y que desperdicia recursos valiosos".

\section{ACTUACIONES RECOMENDADAS}

Es innegable la aportación beneficiosa de la medicina al bienestar de la sociedad y que los avances en este campo contribuyen a mejorar la calidad de vida de las personas. Del mismo modo, la existencia de nuevas demandas es un reto que exige de nuevas respuestas, pero todo ello no es razón para dejar de reconocer e identificar los problemas que la medicalización puede ocasionar y actuar sobre los mismos.

Los objetivos principales de estas actuaciones deben ser: la no medicalización de la vida (actuaciones preventivas encaminadas a evitar que se desarrolle dicho problema) y la desmedicalización (actuaciones encaminadas a reconducir situaciones ya producidas). Algún autor introduce el término de prevención cuaternaria para hacer referencia al conjunto de actividades sanitarias que atenúan o evitan la medicalización de la vida, especialmente en relación con las intervenciones innecesarias y excesivas del sistema sanitario.

Debe tenerse en cuenta que las recomendaciones de actuación frente a la medicalización de la vida que recoge este documento están basadas en las recomendaciones de expertos que han escrito sobre el tema y en las reflexiones de los integrantes de este grupo de trabajo, y que no existen estudios de intervención que demuestren los resultados obtenidos por su utilización.

La mayoría de las actuaciones propuestas precisan, de forma necesaria, de la participación activa de varios de los actores implicados en el proceso de medicalización (sociedad, políticos, gestores, profesionales, etc.), por lo que no se ha considerado oportuno una división artificial por colectivos y se ha optado por agruparlas en: actuaciones generales, donde la consulta o el sistema sanitario son únicamente una faceta más de su aplicación; actuaciones en el sistema sanitario, donde el nivel político o de gestión del mismo es el principalmente implicado; y actuaciones en consulta, donde la asistencia sanitaria representa el ámbito principal para su aplicación.

\section{Actuaciones generales}

- Delimitación del campo de actuación de la sanidad. La población debe conocer de forma clara qué situaciones corresponden al ámbito 
sanitario y cuáles a otros sectores. Devolver cada problema a su campo correspondiente, además de descargar al sistema sanitario, facilita la resolución de los mismos. No es una tarea sencilla, ya que requiere que la sociedad sufra un cambio en conocimientos, creencias, expectativas, etc. y requiere de tiempo, de unidad de mensaje y la participación de todos los sectores implicados. Se requiere tiempo, ya que todo cambio en creencias o expectativas requiere una adaptación progresiva y nunca puede tratar de imponerse de manera brusca. Se requiere una unidad de mensaje y participación de los distintos sectores implicados; una vez establecidos de forma clara los límites, todos los colectivos implicados deben dar a conocer estos límites y los ámbitos de atención de los problemas excluidos del mismo. De nada sirve que los gestores definan unos límites si los profesionales no los respetan; de nada sirve que los profesionales lancen un mensaje de situaciones excluidas del sistema si los medios de comunicación fomentan consultar al médico por dicho motivo.

- Delimitación de expectativas. La sociedad, además de conocer los problemas a los que el sistema sanitario puede dar respuesta, debe conocer las limitaciones de dicho sistema, en cuanto a la capacidad diagnóstica (accesibilidad, validez...) y terapéutica (eficacia, efectividad...) disponibles para las distintas situaciones patológicas, con el objetivo de no crear falsas expectativas con referencia a las mismas. La sociedad tiene el derecho de saber que la ciencia tiene unas limitaciones y que ni se sabe todo, ni se disponen de pruebas y tratamientos para todo, ni los tratamientos garantizan la curación o resolución del problema. Conseguir que la sociedad conozca las posibilidades y limitaciones del sistema es una tarea que afecta a múltiples actores: los políticos y gestores, definiendo su cartera de servicios y dando a conocer sus limitaciones; los profesionales en su práctica diaria, informando a los pacientes de las limitaciones de las pruebas solicitadas y de los tratamientos prescritos; los medios de comunicación, dando información veraz de las limitaciones diagnósticas y terapéuticas existentes...

- Recuperación de actitudes vitales de la sociedad. La sociedad debe recuperar niveles de tolerancia que se han ido perdiendo y recuperar actitudes como la asunción de lo inevitable, la aceptación de los acontecimientos y de la enfermedad o la aceptación de vivir con incertidumbres. Los políticos, los sociólogos y los demás colectivos implicados deberían realizar un estudio de las causas que han llevado a la sociedad a ese cambio de actitudes, lo que dará las pistas necesarias sobre las actuaciones a implementar para reconducir dicha tendencia. Probablemente, los profesionales, por su lugar privilegiado en este tema, junto a otros actores, como los medios de comunicación, tengan un papel fundamental en estas actuaciones correctoras.

- Promoción de la autonomía de la población. Bajo este epígrafe de fomento de la autonomía de la población se incluyen dos aspectos importantes: autonomía como capacidad de decisión y como capacidad de autocuidados. Promocionar la autonomía de las personas en términos de capacidad de decidir de forma autónoma requiere que previamente estén informadas de forma completa y veraz, conociendo las distintas alternativas posibles y los resultados esperados en cada una de las mismas. Existen estudios que ponen de manifiesto que las personas bien informadas toman decisiones más restrictivas respeto a los tratamientos que los propios profesionales, lo que prueba la validez de esta actuación en el control de la medicalización de la vida. Actualmente, la sociedad tiene acceso directo a la información que, en muchos casos, no es ni veraz ni completa; es más, buena parte de la información proviene, no de los medios de comunicación, sino de los propios usuarios. Estos han asumido en buena parte la función de los antiguos profesionales de la comunicación, por lo que la necesidad de una información veraz probablemente sea hoy, y para el futuro, una entelequia. La promoción de los autocuidados juega igualmente un papel importante en la desmedicalización de la sociedad. La mayoría de los recursos sanitarios y los más cercanos al paciente están fuera del sistema sanitario (son los formados por el propio individuo, su familia, su entorno social...), y además muchos problemas médicos, y también no médicos, pueden ser solucionados de forma adecuada por la persona y su entorno. La capacidad de autocuidados aumenta si ayudamos a que sepan convivir con la incertidumbre. Un aspecto a destacar en este apartado es la automedicación. Por épocas denostada, la automedicación "per se" no es negativa; tiene aspectos positivos y debe estimularse para el abordaje de cuadros menores. Como en las actuaciones comentadas anteriormente, son distintos los actores implicados: autoridades sanitarias, profesionales, farmacéuticos, medios de comunicación, etc. 
- Implicación de los medios de comunicación. Aunque ya han sido mencionados en las distintas actuaciones anteriores, su importancia hace que sea necesario dedicarles un apartado específico. Ya que en el ámbito de la sociedad se generan la mayoría de creencias y expectativas, la complicidad de los medios de comunicación es fundamental para evitar la medicalización de la vida, fomentado la no medicalización, destruyendo mitos, promoviendo el empleo de intervenciones eficaces, etc. Algunas de las grandes críticas que reciben los medios de comunicación son la falta de verificación de algunas noticias, los sesgos en la importación por su tendencia a las noticias sensacionalistas y la existencia ocasional de conflictos de intereses. Para evitar estos problemas, los medios de comunicación deberían tomar medidas; a modo de ejemplo se pueden mencionar los Manuales de Ética de algunos periódicos como el New York Times o las recomendaciones de la ONG "Media Doctor" (http://www.mediadoctor.org.au).

\section{Actuaciones en el sistema sanitario}

- Delimitación del campo de actuación de la sanidad. Los políticos y gestores de la sanidad son los responsables principales de la definición de los servicios prestados por el sistema sanitario. La determinación de la cartera de servicios debe basarse en necesidades reales (no en demandas) y en criterios científicos (evidencia). Las Sociedades Científicas deben colaborar en esta determinación. Esta delimitación, como se ha comentado ampliamente en el apartado anterior, debe ser posteriormente dada a conocer a la sociedad.

- Promoción de los valores de la medicina. Otra herramienta de lucha frente a la medicalización de la vida es el fomento y defensa de los valores básicos de la medicina, que compaginan el componente técnico con el componente humano, sin olvidar los aspectos éticos que la impregnan. Esta labor es tanto de los profesionales como de los políticos-gestores, sin olvidar la contribución que pueden hacer los medios de comunicación.

- Atención Primaria como eje del sistema. Las características definitorias de la Atención Primaria (integral, integrada, longitudinal...) la convierten en el ámbito idóneo de atención para la lucha contra la medicalización. El sistema debe estructurarse para permitir el máximo desarrollo de las competencias de la medicina de familia, que permita una atención integral e integrada y que evite la fragmentación de la misma, la sobretecnificación de la atención, etc. Pero para que ello pueda desarrollarse, se requiere de la solución de viejas demandas de este nivel asistencial: cupos limitados que permitan dedicar un mínimo de tiempo a cada paciente, desburocratización de la atención, acceso a métodos diagnósticos que eviten derivaciones innecesarias, etc.

- Dimensionar correctamente la medicina preventiva. La medicina preventiva tiene un indudable valor, pero debe evitarse la puesta en marcha de actividades preventivas no basadas en evidencias y que únicamente consiguen considerar como enfermos a personas sanas. Ante un riesgo, únicamente debería intervenirse si dicho riesgo puede tener un impacto real sobre las personas y cuando además se disponga de intervenciones eficaces y seguras para controlarlo. En definitiva, se trataría de prevenir la prevención innecesaria.

- Desburocratización sanitaria de la vida. Una forma de evitar la medicalización de la vida es sacar de la atención sanitaria contactos burocráticos de la población con el sistema por motivos ajenos a las funciones del mismo. Sirvan como ejemplos los partes de descanso maternal (no se certifica una enfermedad, únicamente se constata una situación para acceder a un derecho laboral), los informes para las vacaciones de los servicios sociales (donde su salud cuenta menos que sus ingresos o si ya ha disfrutado del servicio los años previos) o las notas por falta de asistencia al colegio de un niño (los padres son los responsables y debería ser suficiente con su notificación evitando la consulta por un cuadro banal).

- Promoción de la investigación independiente. $\mathrm{Si}$ algunos de los problemas comentados son la falta de evidencia de cómo actuar ante multitud de situaciones y el sesgo existente en la investigación realizada por la influencia de la industria farmacéutica, parece evidente que los servicios sanitarios, apoyándose en organizaciones como las Sociedades Científicas, deberían liderar la investigación dando prioridad a las situaciones donde existan lagunas en el conocimiento y en la evaluación de la efectividad de las intervenciones.

- Promoción de la formación. Una correcta atención, basada en la toma de decisiones que se apoyen en las evidencias, solo puede hacerse por profesionales correctamente formados. Las 
Instituciones Sanitarias deben liderar la formación de los profesionales que las integran con el objetivo de dar una formación independiente y de calidad que permita a los profesionales una correcta actualización de sus conocimientos.

- Regulación y control de las actuaciones de la industria farmacéutica. Se debe regular y controlar tanto la investigación como la aprobación de nuevos productos, a través de agencias independientes (la FDA y la EMEA deben disipar las sombras de duda existentes por su relación con la industria). Del mismo modo, debe existir un control riguroso de la promoción tanto directa como indirecta que se realiza de sus productos, y tanto a nivel de los profesionales como de la población general.

\section{Actuaciones en consulta}

- Visión integral e integrada del paciente. La visión integral del paciente con todos sus problemas evita la sobreactuación (más visitas, más pruebas diagnósticas y más tratamientos) que la fragmentación de la atención genera. La visión integrada permite la exploración y aprovechamiento de sus conocimientos, de sus expectativas e incertidumbres, de los recursos informales disponibles, etc.

- Capacidad resolutiva. Tener capacidad resolutiva hace referencia a dos aspectos diferentes pero complementarios: acceso a los recursos y respuesta a las demandas. El acceso a los recursos del sistema que permita el estudio y tratamiento de los procesos ya ha sido comentado en el apartado de actuaciones a nivel sanitario. La capacidad resolutiva, como respuesta a las demandas, hace referencia a que toda demanda debe encontrar una respuesta adecuada, sin que ello signifique que cada demanda precise de un tratamiento.

- Actuación acorde a las evidencias científicas existentes. Ello conlleva, en primer lugar, que no hay que estudiar/tratar lo que no requiere estudio/ tratamiento o para lo que no existe estudio/ tratamiento. Por otra parte, al hablar de trabajar con evidencia científica, no debe confundirse significación estadística con relevancia clínica, por lo que es preciso valorar la calidad de las investigaciones en las que se basa dicha evidencia y, además, tener en cuenta aspectos como la utilidad y el beneficio. El seguimiento de recomendaciones, guías de práctica clínica, etc. facilita esta forma de trabajar. Debe seguirse la máxima de que toda actuación no fundamentada es iatrogénica (médica, social o culturalmente).
- Racionalización en la utilización de los recursos. Muy en relación con el trabajo basado en la evidencia científica, no debe confundirse el acceso a los recursos diagnósticos y terapéuticos (demandado en este documento) con uso irracional de los mismos. Los criterios de utilización se deben basar en conceptos como utilidad, eficacia/efectividad o beneficio, pero nunca en las demandas de la población o en la medicina defensiva.

- Estructuración desmedicalizadora de la consulta. Una correcta realización de la entrevista clínica contribuye a dificultar la medicalización de las situaciones, al intervenir sobre varios de los factores causales de la misma (regulación de expectativas, promoción de autocuidados, manejo de la incertidumbre, etc.), y ello puede realizarse en sus distintas fases:

1. Escucha activa. Permite clarificar y entender la verdadera demanda y las expectativas del paciente (que vienen influidas por sus creencias, su entorno...). Esta escucha activa facilita, además, crear una relación que permite descargar parte de la ansiedad generada por la existencia del problema y tiene función terapéutica.

2. Formulación de hipótesis. Posibilita un nuevo enfoque del problema que facilita la reflexión (por ejemplo: ¿cree que lo que siente puede tener alguna relación con su problema en el trabajo?) y el autocuidado (por ejemplo: ¿cree que puede ayudarle si habla del tema con su familia?).

3. Reconocimiento de la normalidad. Explicación de los criterios de normalidad o enfermedad y de las variantes de la normalidad, significado de los síntomas y/o signos y de la necesidad o no de tratamiento y de controles periódicos.

4. Reconversión de creencias y ajuste de expectativas. Dado que los profesionales sanitarios somos una de las principales fuentes de información que generan estas creencias y expectativas, se debe actuar sobre las mismas: dando información sobre la capacidad real del sistema en general y de nuestra capacidad individual, informando sobre el origen y el curso natural de la enfermedad, posibilidades terapéuticas y pronóstico, etc.

5. Trabajar la tolerancia a la incertidumbre. Aunque parezca un contrasentido, conocer 
la incertidumbre en nuestro trabajo permite trabajar con mayor seguridad. Del mismo modo, se debe hacer participe al paciente de dicha incertidumbre para facilitar la adecuación de las expectativas, reconducir demandas, etc. Todo ello se consigue a través de la vigilancia de los procesos, del conocimiento y explicación del curso natural de las enfermedades, de los signos y síntomas de alarma, de la utilización en las primeras fases de la enfermedad de tratamientos no farmacológicos, etc.

6. Redirigir la demanda. Se deben evitar las actuaciones médicas por problemas no médicos y la mejor forma de realizarlo es a través de la verbalización de esta situación y la derivación del problema al ámbito adecuado para su resolución. Ello no implica no escuchar; significa escucha empática del problema, pero derivación firme del mismo.

7. Compartir decisiones. La aceptación y la responsabilización de nuestras actuaciones por parte del paciente mejora los resultados de la misma tanto en términos de resultados como de satisfacción, pero ello requiere el aporte de información real y no sesgada, y de la toma de decisiones compartidas.

8. Tratamiento. Es cierto que cada demanda a consulta requiere de una respuesta, pero dicha respuesta no siempre es preciso que sea prescribir un fármaco, aunque exista tendencia a ello; la prescripción debe basarse en las evidencias existentes y en el razonamiento clínico. El profesional que prescribe es el responsable de la prescripción; debe gestionarse de forma activa para evitar la prescripción inducida.

- Formación Continuada. Aunque el sistema sanitario debe facilitar el acceso a la formación, los responsables últimos a nivel individual son los propios profesionales.

A modo de reflexión final de todas estas recomendaciones, se pueden mencionar las reflexiones expuestas por Smith en un editorial de 2001 aparecido en la revista British Medical Journal:

- La población debe aceptar que la enfermedad, el dolor y la muerte son parte de la vida.

- La población no debe delegar sus problemas en los profesionales.

- La medicina tiene una capacidad limitada, especialmente en problemas sociales, y además tiene riesgo.
- Los profesionales deben conocer sus limitaciones.

- Los políticos y gestores deben centrarse en la realidad y evitar crear expectativas irreales.

\section{BIBLIOGRAFÍA}

1. Angell M. Excess in the pharmaceutical industry. CMAJ. 2004; 171:1451-53.

2. Blech J. Los inventores de enfermedades. Barcelona: Destino; 2005.

3. Bordas JM, Casajuana J. Prescripción inducida en atención primaria: de la excusa pasiva a la gestión activa. FMC. 2000; 5:323-28.

4. Cabral JA. Nuevas tendencias de la medicalización. Ciencia \& Saude Colectiva. 2008; 13(supl):579-87.

5. Campbell EG, Russell LG, Mountford J, Miller LG, Cleary $\mathrm{PD}$, Blumenthal $\mathrm{D}$. A national survey of physician-industry relationships. N Engl J Med. 2007; 356:1742-50.

6. Campbell EG, Weissman JS, Ehringhaus S, Rao SR, Moy $B$, Goold SD. Institutional Academic-Industry relationship. JAMA. 2007; 298:1779-86.

7. Campbell EJM, Scadding JG, Roberts RS. The concept of disease. BMJ. 1979; 8:757-62.

8. Casino G. La Promoción de la Enfermedad. El País. 2006 (25/04/06). p. 39.

9. Davis P. Health care as a risk factor. CMAJ. 2004; 170:168889.

10. Díaz JL. El sufrimiento medicalizado. Cultura de los cuidados. 2008; 23:50-6.

11. Fernández $M J$, Marquet $R$, Reguant $M$, Zaapter $F$. ¿Enfermos de salud?. Societat Catalana de Medicina Familiar i Comunitária. 2006. Disponible en: http://www. camfic.org.

12. Gérvas J. Malicia Sanitaria y prevención cuaternaria. Gac Med Bilbao. 2007; 104:93-6.

13. Gérvas J, Pérez-Fernández M. El auge de las enfermedades imaginarias. FMC. 2006; 13:109-11.

14. Gérvas J. Moderación en la actividad médica preventiva y curativa. Cuatro ejemplos de necesidad de prevención cuaternaria en España. Gac Sanit. 2006; 20(supl 1):127-34.

15. Gottlieb S. A fith of Americans contact their doctor as a result of drug advertising. BMJ. 2002; 325:854.

16. Grilli R, Ramsay C, Minozzi S. Mass media interventions: effects on health services utilisation (Cochrane Review). In: The Cochrane Library. Inssue 4, 2002. Oxford: Update software.

17. Hijmans E, Pleijter A, Wester F. Covering Scientific Research in Dutch Newspapers. Science Comunication. 2003; 25(2):153-76.

18. Hofmann B. The technological invention of disease. J Med Ethics: Medical Humanities. 2001; 27:10-9.

19. Illich J. L'obsesion de la santé parfacte. Le Monde Diplomatique. 1999; 41:21.

20. Induces prescription in Barcelona's Primary Care Study Group (Institut Catalá de la Salut). Induced prescription in primary healthcare. Eur J Gen Pract. 1999; 5:49-53.

21. Kishore J. A dictionary of Public Health. New Dephi: Century Publications; 2002.

22. Lumbreras B, Hernández-Aguado I. El entusiasmo por las pruebas diagnósticas: efectos en la salud y formas de control. Gac Sanit. 2008; 22(Supl 1):216-22.

23. Mainetti JA. La Medicalización de la Vida. Electroneurobiología 2006; 14:71-89.

24. Maglio I. La medicalización de la vida como causa de reclamos contra la práctica médica. Medicina (Buenos Aires). 1998; 58: 548-53.

25. Marquet R. La obsesión por la salud. FMC. 2006; 13:507-9.

26. Márquez-Calderón $\mathrm{S}$. Los efectos negativos de las intervenciones preventivas basadas en el individuo. Gac Sanit. 2008; 22(Supl 1):205-15. 
27. Marquez S, Meneu R. La medicalización de la vida y sus protagonistas. Ges Clinica y Sanitaria. 2003; 5:47-53.

28. Meador $\mathrm{C}$. The art and science of nondisease. N Engl J Med. 1965; 272:92-5

29. Meneu R. Las "no enfermedades". AMF. 2005; 1:123-4.

30. Merino A, Miguel F, Sanz R. Yo medicalizo, tú medicalizas, él...; reflexiones y propuestas. AMF. 2005; 1:269-78.

31. Miguel F, Merino A, Sanz R, García A, Montero MJ, Maderuelo JA. Procedimientos operativos y técnicos en la medicalización de la prevención. Aten Primaria. 2009. 41:702-6.

32. Moynihan R, Doran E, Henry D. Disease mongering is now part of the global health debate. Plos Med. 2008; 5:e106.

33. Moynihan R, Heath I, Henry D. Selling sickness: the pharmaceutical industry and disease mongering. BMJ. 2002; 324:886-91

34. New York Times. Ethical Journalism: a handbook of values and practices for the News and Editorial Departaments. New York; 2004.

35. Pellechia M. Trenes in science coverage: a contect análysis of three US newspapers. Public Understanding of Science. 1997; 6:49-68.

36. Puig-Junoy J. La financiación y la regulación del precio de los medicamentos en el Sistema Nacional de Salud: cambios y continuidad. Gac Sanit. 2007; 21(1):1-4.

37. Pundik J. La medicalización de la infancia. ¿Cada vez más frecuente? FMC. 2009; 16:213-18.

38. Quintana O. Los objetivos de la medicina. Rev Calidad Asistencial. 2003; 18:132-35.

39. Reverte D. El negocio de la sanidad y la medicalización de la vida. Salud 2000. 2007; 112:8-16.
40. Rodríguez S. El proceso de medicalización y sus consecuencias. Entre la moral, el poder y el negocio. Intersticios. 2008; 2:71-85.

41. Schwitzer G, Mudur G, Henry D, Wilson A, Goozner M, Simbra $M$ et al. What are the roles and responsibilities of the media in disseminatinh health information? Plos Med. 2005; 2(7):e215.

42. Sen A. Health: perception versus observation. BMJ. 2002; 324: 860-1.

43. Smith R. Why are doctors so unhappy? There are probably many causes, some of them deep. BMJ. 2001; 322:107374.

44. Smith R. In search of "non-disease". BMJ. 2002; 324:883-5.

45. Sociedad Castellano-Manchega de Medicina Familiar y Comunitaria. Nuevas demandas de la población. Scamfyc. 2005. Disponible en: http://www.scamfyc.org.

46. Sociedad Castellano-Manchega de Medicina Familiar y Comunitaria. Propuestas para mejorar el uso racional del medicamento y racionalizar el gasto farmacéutico. Scamfyc. 2003. Disponible en: http://www.scamfyc.org.

47. Tizon JL. De los procesos de duelo a la medicalización de la vida. Jano. 2006; 1818:58-60.

48. Vara AM. Como medicalizar la vida diaria: la creación de enfermedades o "disease mongering". Evid Actual Pract Amb. 2008; 11:130-2.

49. Zapater F. Hasta aquí hemos llegado. Los límites de la medicina. Rev Calidad Asistencial. 2006; 21:1-2.

50. Zapater $F$. nuevos pacientes y nuevas demandas. ¿Qué hacer? En: Casajuana J. Gestión del día a día en el Equipo de Atención Primaria. Barcelona: SemFYC; 2007

51. Zapater F, Vila R. Habilidades para no medicalizar. FMC. 2008; 15:670-2. 